\title{
Evidence of Impaired Night-Response of Blood Pressure in Hypertensive Smokers. An Update*
}

\begin{abstract}
Epidemiological survey has demonstrated an impaired night response of BP in hypertensive smokers.

This Ambulatory Blood Pressure Monitoring Study (ABPM) conducted in hypertensive chronic smokers and updated by the analysis of more recent findings undoubtedly shows that a large majority of these individuals belongs to the group of non-dipper with a nocturnal fall in BP more than $10 \%$.
\end{abstract}

Keywords: Smoking; Hypertension; Non-dippers; Dippers

\section{Research Article}

Volume 4 Issue 2 - 2015

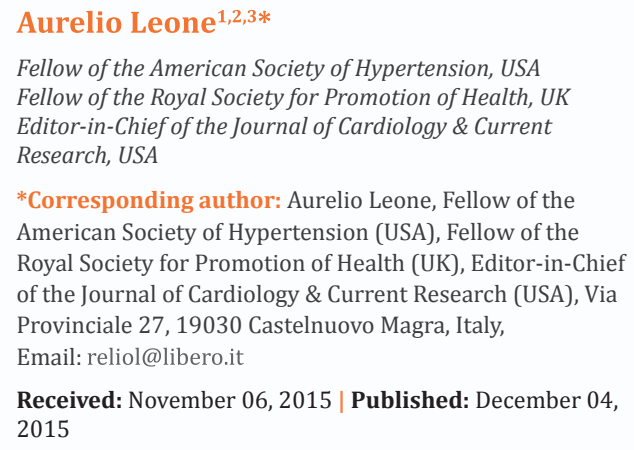

*Corresponding author: Aurelio Leone, Fellow of the American Society of Hypertension (USA), Fellow of the Royal Society for Promotion of Health (UK), Editor-in-Chief of the Journal of Cardiology \& Current Research (USA), Via Provinciale 27, 19030 Castelnuovo Magra, Italy, Email: reliol@libero.it

Received: November 06, 2015 | Published: December 04, 2015

Abbreviations: S: Smoking; H: Hypertension; BP: Blood Pressure; ABPM: Ambulatory Blood Pressure Monitoring

\section{Introduction}

Smoking (S) and hypertension $(\mathrm{H})$ are major risk factors for cardiovascular disease [1-3]. A crucial question is to assess whether $\mathrm{S}$ favors the appearance of $\mathrm{H}$ and potentiates the complications associated with hypertensive disease. There is evidence that blood pressure (BP) follows a circadian rhythm [4] with morning increase and nocturnal fall in its values. Two groups of hypertensive patients have been described: dipper subjects, who have nocturnal blood pressure lowering more than $10 \%$, and non-dipper individuals with a nocturnal fall in blood pressure less than $10 \%$. The non-dipper hypertensive patients are believed to be at an increased risk of cardiovascular events [5]. The purpose of this study was to update our previous data on the response to 24-hour Ambulatory Blood Pressure Monitoring (ABPM) of hypertensive out-patient smokers, enrolled from the Department of Internal Medicine, University of Pisa (year 2010), and Department of Internal Medicine, City Hospital Massa, Italy (data from the year 1999 to 2002) investigated in a previous report partly presented at the 2011 Meeting of the American Society of Hypertension in New York City [6].

\section{Material and Methods}

Study material (Table 1) consisted of 104 hypertensive men, aged from 55 to 72 years (mean: 63+/-8 years), 72 smokers (69\%) and 32 non-smokers (31\%), used as a control group, undergone 24-hour ABPM. Mean day systolic BP was 162 (+/18) $\mathrm{mmHg}$ and diastolic BP $92+/-7 \mathrm{mmHg}$ for smokers group and 159+/-17 mmHg for systolic BP and 94+/-6 mmHg for diastolic BP in non-smoker control group. Statistical analysis was conducted by using paired T-test, with a $\mathrm{P}<0.05$ significant.

\section{Results}

48 hypertensive smokers (67\%) were non-dippers for systolic BP (night BP falls from 0 to $7 \%$ ) while the other 33\% (24 patients) were dippers with a BP fall from 10 to 13\% (Table 2). Diastolic reduction was from 3 to $8 \%$ in 39 smoker patients (54\%) and from 12 to $14 \%$ in others. On the contrary, among hypertensive non-smokers (Table 3), there were 26 dippers (81\%) for systolic BP with a nightfall from 12 to $15 \%$ and 24 (75\%) for diastolic BP (reduction from 10 to 14\%), and 6 (19\%) non-dippers with a systolic and diastolic night-BP fall from 4 to $9 \%$. Statistical difference (P less than 0.05) existed between non-dipper smokers and dipper non-smokers as well as between non-dipper and dipper smokers particularly for systolic BP values.

Table 1: Characteristics of Study Population.

Patients (number) 104 (100\%)

Sex (male) $104(100 \%)$

Age (mean year+/-SD) 63+/-8

Smokers 72 (69\%)

Non-smokers (control group) 32 (31\%)

ABPM 104 (100\%)

Mean systolic BP (mmHg, smokers) 162+/-18

Mean diastolic BP (mmHg, smokers) $92+/-7$

Mean systolic BP (mmHg, non-smokers) 159+/-17

Mean diastolic BP (mmHg, non-smokers) 94 +/-6 
Table 2: Night-response in smokers for systolic and diastolic BP.

\begin{tabular}{|c|}
\hline BP Night fall(dippers) Night fall(non-dippers) \\
$\mathrm{N}^{\circ} \mathrm{N}^{\circ}$ \\
\hline Systolic BP $24(33 \%)<0-7 \% 48(67 \%)>10 \%$ \\
Diastolic BP $33(46 \%) 3-8 \% 39(54 \%)>10 \%$ \\
Total 5787 \\
\hline
\end{tabular}

Table 3: Non-smoker dippers and non-dippers.

BP Night-fall(dippers) Night fall(non-dippers)

$\%$ fall BP $\mathrm{N}^{\circ} \%$ fall BP $\mathrm{N}^{\circ}$

Systolic BP 12-15\% 26(81\%) 4-9\% 6 (19\%)

Diastolic BP 10-14\% 24(75\%) 4-9\% 6 (25\%)

Total 5012

\section{Conclusion}

In this study, hypertensive smokers were more frequently non-dippers particularly for systolic values of BP. It is worth noting that this pattern usually identifies a major extent of cardiovascular damage caused by the association of $\mathrm{S}$ with $\mathrm{H}$. Moreover, non-dipper smokers usually have a night reduced fall in BP than that seen in non-dipper non-smokers (Figure 1). Previous and following papers $[7,8]$ to the observations here described achieved similar results, although with little differences in the methods of study and parameters assessed. The primary pathological mechanism to explain these responses is related to the role of adrenergic and sympathetic system, which play a different activity on BP in the morning and night. Thus, it is worth noting that $\mathrm{S}$ strongly feels the effects of the last two parameters, which are influenced by nicotine and carbon monoxide [9]. Therefore, the adverse relationship between S and BP cannot be absolutely denied as this finding undoubtedly shows

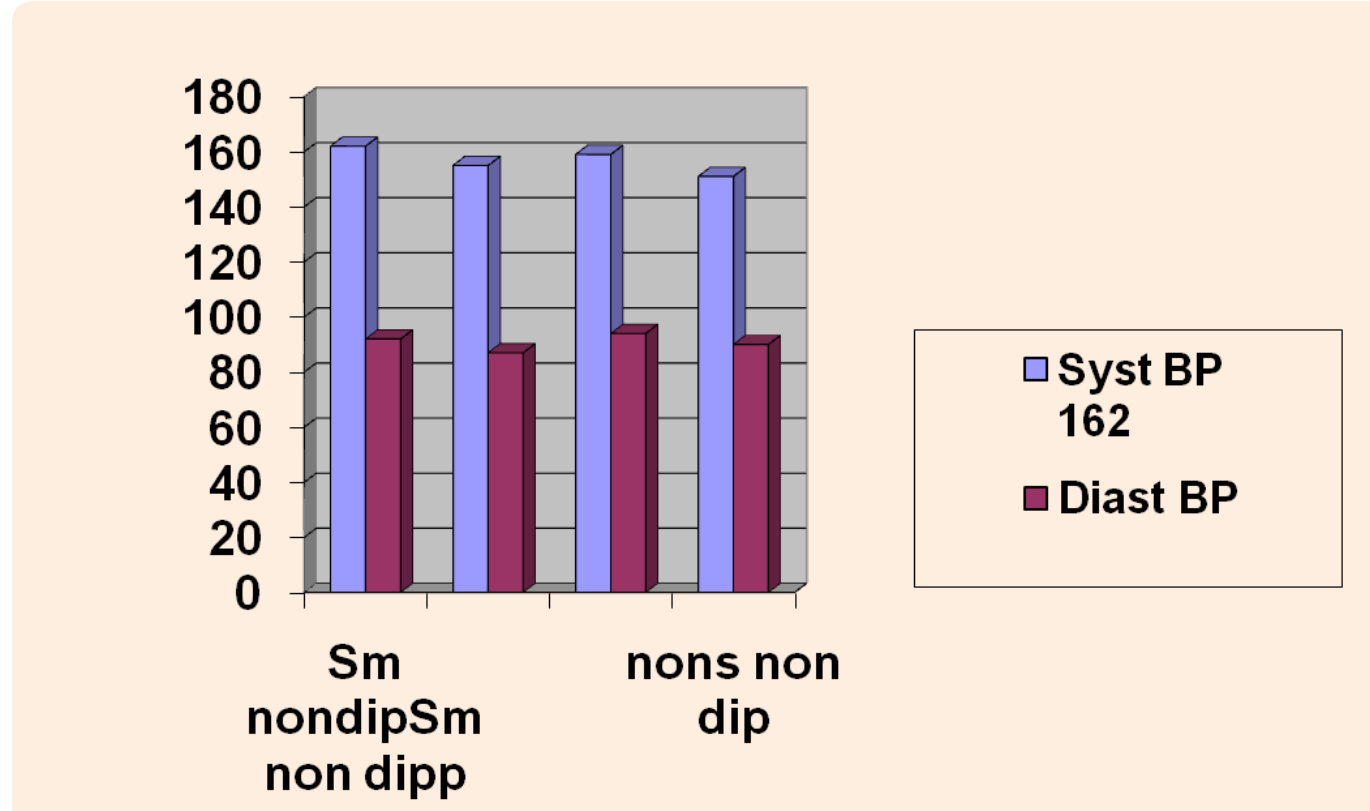

Figure 1: Day-night BP in non-dipper smokers and non-dipper non-smokers. Although all hypertensive subjects were non-dippers, there is evidence that smokers have a reduced fall in night BP compared to non-smokers. A: Non-dipper smokers day BP; B: Non-dipper smokers night BP; C: Non dipper non-smokers day BP; D: Non-dipper non-smokers night BP.

\section{References}

1. Hopkins PN, Williams RR (1986) Identification and relative weight of cardiovascular risk factors. Cardiol Clin 4(1): 3-31.

2. Leone A (2003) Relationship between cigarette smoking and other coronary risk factors in atherosclerosis: risk of cardiovascular disease and preventive measures. Curr Pharm Des 9(29): 2417-2423.

3. Leone A, Landini L, Leone A (2010) What is tobacco smoke? Sociocultural dimensions of the association with cardiovascular risk. Curr Pharm Des 16(23): 2510-2517.
4. Uzu T, Kimura G (1999) Diuretics shift circadian rhythm of blood pressure from nondipper to dipper in essential hypertension. Circulation 100(15): 1635-1638.

5. Fagard RH, Celis H, Thijs L, Staessen JA, Clement DL et al. (2007) Daytime and nighttime blood pressure as predictors of death and cause-specific cardiovascular events in hypertension. Hypertension 51(1): 55-61.

6. Landini L, Leone A (2011) Ambulatory Blood Pressure Monitoring in Hypertensive Smokers: Evidence of Impaired Night-Response. JCH 289. 
7. Li H, Srinivasan S, Berenson GS (2006) Comparison of the measures smokers and former smokers: the Bogalusa Heart Study. Am J Hypertens 19(9): 897-901.

8. Rymkiewicz E, Rekas-Wòjcik A, Bukszynska-Soldaj K, Dzida G O
Coexistence of non-dipper hypertension with other chronic disease. Arterial Hypertens 19(2): 84-87.

9. Leone A (2014) Endothelial dysfunction in passive smokers. J Cardiol Curr Res 1(7): 00039 